\title{
Influence of the operating conditions on highly oxidative radicals generation in Fenton's systems
}

\author{
Cesare Ciotti ${ }^{\mathrm{a}}$, Renato Baciocchi ${ }^{\mathrm{a}, *}$, Tuula Tuhkanen ${ }^{\mathrm{b}}$ \\ a Department of Civil Engineering, University of Rome "Tor Vergata", Via del Politecnico 1, 00133 Rome, Italy \\ ${ }^{\mathrm{b}}$ Department of Environmental Engineering and Biotechnology, Tampere University of Technology, P.O. Box 541, 33101 Tampere, Finland
}

\section{A R T I C L E I N F O}

\section{Article history:}

Received 9 July 2007

Received in revised form 26 March 2008

Accepted 27 March 2008

Available online 8 April 2008

\section{Keywords:}

Oxidative radicals

Fenton's system

Rotatable Central Composite

Soil

\begin{abstract}
A B S T R A C T
In this work, an indirect method for estimating the total amount and concentration of oxidative radicals in aqueous and slurry-phase Fenton's systems was developed. This method, based on the use of benzoic acid as probe compound, was applied for evaluating the effect of the operating conditions on the radicals amount produced, their production efficiency (i.e. moles of radicals generated per mole $\mathrm{H}_{2} \mathrm{O}_{2}$ ) and their concentration. A Rotatable Central Composite design (RCC) was used to select the operating conditions in order to get a statistically meaningful data set. Hydrogen peroxide and ferrous ion concentrations ranged between $0.2-1 \mathrm{mM}$ and $0.2-0.5 \mathrm{mM}$, respectively; humic acid concentration between 0 and $15 \mathrm{mg} / \mathrm{L}$, whereas the soil/water weight ratio in slurry-phase systems between 1:10 and 9:10. The probe compound concentration was 9 or $0.1 \mathrm{mM}$ in experiments aimed to evaluate the total amount or concentration of oxidative radicals, respectively. The obtained results indicated that the amount of radicals generated in both aqueous and soil slurry Fenton's system increased with higher $\mathrm{H}_{2} \mathrm{O}_{2}$ concentration and, more specifically, that their production efficiency increased with increasing $\mathrm{Fe}(\mathrm{II}): \mathrm{H}_{2} \mathrm{O}_{2}$ molar ratio. Addition of dissolved organic compounds as humic acid did not notably affect the oxidative radicals amount and concentration. On the contrary, a one order of magnitude reduction in both radicals amount generated and concentration was observed when soil was added to the reaction environment.
\end{abstract}

(c) 2008 Elsevier B.V. All rights reserved.

\section{Introduction}

Fenton's reaction is widely used for the remediation of contaminated soil and groundwater, with a large number of in situ applications. The chemistry of the Fenton's process is based upon the reaction of hydrogen peroxide $\left(E^{0}=1.80\right.$ and $0.87 \mathrm{~V}$ at $\mathrm{pH} 0$ and 14 respectively) with a proper catalyst, leading to the generation of a pool of radicals [1], capable of non-selectively oxidizing a wide range of biorefractory organic pollutants such as chlorinated aliphatics, halogenated phenols, PAHs and PCBs. The radical produced in the Fenton's initiation Reaction (I) is the hydroxyl radical, whose formation can be achieved by adding an homogeneous catalyst, such as a transition metal salt $[3,4]$, by generating the metal catalyst electrochemically by means of sacrificial steel electrodes or by using the metals naturally occurring in the environment as heterogeneous catalyst [5-12]:

$$
\mathrm{H}_{2} \mathrm{O}_{2}+\mathrm{Fe}^{2+} \rightarrow \mathrm{OH}^{\bullet}+\mathrm{OH}^{-}+\mathrm{Fe}^{3+}
$$

\footnotetext{
* Corresponding author. Tel.: +39 0672597022 (7039); fax: +39 0672597021 E-mail address: baciocchi@ing.uniroma2.it (R. Baciocchi).
}

The hydroxyl radicals generated through Reaction (I) react with hydrogen peroxide and begin a series of propagation reactions [2]:

$$
\begin{aligned}
& \mathrm{OH}^{\bullet}+\mathrm{H}_{2} \mathrm{O}_{2} \rightarrow \mathrm{HO}_{2}^{\bullet}+\mathrm{H}_{2} \mathrm{O} \\
& \mathrm{HO}_{2}^{\bullet} \rightarrow \mathrm{O}_{2}^{\bullet-}+\mathrm{H}^{+} \\
& \mathrm{HO}_{2}^{\bullet}+\mathrm{Fe}^{2+} \rightarrow \mathrm{HO}_{2}^{-}+\mathrm{Fe}^{3+}
\end{aligned}
$$

These propagation reactions produce a pool of radicals with different reactive properties. Namely, hydroxyl radical $\left(\mathrm{OH}^{\bullet}\right)$ and perhydroxyl radical $\left(\mathrm{HO}_{2}{ }^{\bullet}\right)$ are both oxidant, although the latter is a relatively weak one; superoxide radical $\left(\mathrm{O}_{2}{ }^{\bullet-}\right)$ is a weak reductant and nucleophile in aqueous systems [2]. The oxidative properties of a Fenton's system is therefore the result of the combined activities of the different radical generated and is not strictly correlated to just one radical species. This consideration becomes more and more valid as the hydrogen peroxide concentration is increased, when Reaction (III) is more important [2].

Even though the Fenton's process is considered in many instances as a mature technology, with hundreds of pilot-scale and full-scale applications, mainly concentrated in the United States, the design of the operating conditions (oxidant dosage, optimal amendments concentration, etc.) is still based on the evaluation of the contaminant removal efficiency, obtained through long and 
time-consuming lab-scale and pilot-scale feasibility tests. Recently, the possibility of using the hydrogen peroxide lifetime as an indicator of the oxidation efficiency of Fenton's and Fenton-like processes has been proposed to simplify at least the first screening phase of the experimental design activity and for selecting, among the different operating conditions, those which may be potentially effective for the oxidation treatment $[13,14]$. The obtained results demonstrated that the procedure was successful for comparing the different Fenton's process operating conditions for the same soil, but failed when applied to make a comparison between different soils, meaning that the intrinsic soil characteristics (structure, presence of both organic and inorganic substances) may modify both hydrogen peroxide stability and radicals activity with respect to the target compounds. Even though the presence of hydrogen peroxide in the reaction environment is somehow a prerequisite for the formation of oxidative radicals, its concentration is not necessarily related to the radicals one. A direct measurement of these radicals would allow to assess the influence of the operating conditions on the effectiveness of the Fenton's reaction. Unfortunately, the methods applied so far (i.e. electron spin resonance spectroscopy (ESR)) result unsuitable for being applied as routine procedures, due to their high-cost and time-consuming features. On the contrary, an indirect method for radical quantification looks more attractive for this kind of applications. The method hereby proposed is based upon the quantification of the products generated by the reaction between oxidative radicals and an organic probe compound, which is used as a radical scavenger. The probe compound should be characterized by few and very stable reaction products and by a well-known and established reaction constant with reactive radicals. Dimethyl sulfoxide [15], salicylic acid [16], phenols or pyrocathecols [17] are among the most commonly used compounds. Recently, Lindsey and Tarr [18] selected benzoic acid and $n$-propanol as chemical probes to quantify the hydroxyl radicals production in Fenton's systems, allowing to measure both hydroxyl radical formation rate and concentration. Different systems such as pure water, aqueous solutions of fulvic acid or humic acid and natural surface waters were tested in order to determine the influence of organic substrates on hydroxyl radicals production. The reactions between hydroxyl radicals and probe compounds were performed under controlled conditions: high probe concentrations were applied to determine the radicals total amount, while low probe concentrations were used to measure the hydroxyl radicals concentration. A linear correlation between hydrogen peroxide concentration and hydroxyl radicals formation was found, while the presence of organic substrates was observed to induce an up to 4 -fold reduction of $\mathrm{OH}^{\bullet}$ formation rate and $\mathrm{OH}^{\bullet}$ concentration with respect to pure water systems. The Lindsey method addresses pro forma the $\mathrm{OH}^{\bullet}$ radical but it really refers to the sum of the highly oxidative radicals generated by the Fenton's reagent in solution. Therefore, this method will be applied in this paper for the quantification of oxidative radicals generated in a Fenton's system with two main goals. On the one hand, we want to apply the Lindsey method for evaluating the influence of the operating conditions on the oxidative radicals produced during the Fenton's reaction. On the other hand, the paper is also aimed to assess the feasibility of the Lindsey method for soil slurry systems. To this purpose, experiments in aqueous phase (with and without humic acid) and in soil slurry phase are presented and discussed. The selection of the operating conditions is performed using a Rotatable Central Composite (RCC) method, in order to minimize the number of meaningful experiments to be performed and to obtain a correct evaluation of the influence of the operating conditions on the process performances. These were evaluated by measuring the total amount of generated radicals, their concentration and their production efficiency (i.e. the fraction of $\mathrm{H}_{2} \mathrm{O}_{2}$ converted to oxidative radicals).

\section{Experimental}

\subsection{Materials}

Benzoic acid (BA), p-hydroxybenzoic acid ( $p$-HBA), hydrogen peroxide $\left(\mathrm{H}_{2} \mathrm{O}_{2}, 30 \%, \mathrm{w} / \mathrm{w}\right.$ solution), iron(II) sulphate heptahydrate, hydrochloric acid, 1-propanol ( $\mathrm{PrOH})$, sodium monobasic phosphate, sodium hydroxide and acetonitrile were purchased by Sigma-Aldrich (HPLC-grade or ACS-grade). Humic acid sodium salt (HA) (technical grade, $50-60 \%$ as humic acid) was purchased by Acros Organics. All materials were used as received. Ultra-pure water was provided by a Millipore Milli-Q Plus generator.

\subsection{Method for oxidative radicals detection}

The total amount of generated oxidative radicals and their concentration were evaluated in both aqueous solution and soil slurry phase by using the approach developed by Lindsey and Tarr [18]. The Lindsey method addresses pro forma the $\mathrm{OH}^{\bullet}$ radical but it really refers to the sum of the highly oxidative radicals generated by the Fenton's reagent in solution. For sake of simplicity the reactivity of the oxidative radicals was assumed equal to the one of the hydroxyl radicals. This made possible to follow the approach of Lindsey and Tarr [18], who used benzoic acid as probe compound, since its reaction rate constant with hydroxyl radicals is known $\left(K_{\mathrm{BA} / \mathrm{OH}}{ }^{\bullet}=4.2 \times 10^{9} \mathrm{M}^{-1} \mathrm{~s}^{-1}\right)$ as well as the products obtained from their reaction, i.e. $p$-hydroxybenzoic acid ( $p$-HBA), $o$-HBA, $m$-HBA and other products such as ring fission and decarboxylation products (e.g. maleic and oxalic acids, cathecol and hydroquinone [19]). Moreover, the reaction between hydroxyl radicals and benzoic acid is known to require $5.87 \pm 0.18$ radicals moles to produce $1 \mathrm{~mol} p$ HBA [20]. The amount of radicals produced during Fenton's batch scale tests was evaluated assuming that all the generated radicals reacted with BA, thus mainly producing $p$-HBA. This assumption holds true if the fraction of oxidative radicals reacting with $p$-HBA and the other secondary products is negligible with respect to the one reacting with BA, i.e. if the initial BA concentration in solution is large enough. Under this condition, the amount of radicals generated is related to the amount of $p$-HBA produced and can be measured as reported in Section 2.3. The minimum benzoic acid concentration to be used for quantifying the total amount of produced radicals was obtained through preliminary tests, whose results are reported in Fig. 1. It can be noticed that the moles of oxidative radicals trapped increase with the initial benzoic acid concentration, until a threshold BA concentration, over which further increase of the BA concentration does not affect the radical amount anymore. Taking into account the results reported in Fig. 1, all tests for quantifying the total amount of oxidative radicals were performed with a $9 \mathrm{mM}$ BA concentration. Instead, the radicals concentration was estimated assuming that all oxidative radicals react following the same second order rate law of the hydroxyl radicals:

$[\mathrm{Ox}]=\frac{R_{\mathrm{BA}}}{K_{\mathrm{BA} / \mathrm{OH}} \cdot[\mathrm{BA}]}$

where $[\mathrm{Ox}]$ and $[\mathrm{BA}]$ in Eq. (1) are the instantaneous concentrations of oxidative radicals and benzoic acid, respectively. The latter can be determined with the analytical methods reported in Section 2.3. Since the $K_{\mathrm{BA} / \mathrm{OH}}{ }^{\bullet}$ term is known $\left(K_{\mathrm{BA} / \mathrm{OH}}{ }^{\bullet}=4.2 \times 10^{9} \mathrm{M}^{-1} \mathrm{~s}^{-1}\right)$, this method requires simply to evaluate the $\mathrm{BA}$ concentration at different times during a batch Fenton's test and hence the rate of BA oxidation $\left(R_{\mathrm{BA}}\right)$ which, over short time intervals can be evaluated 


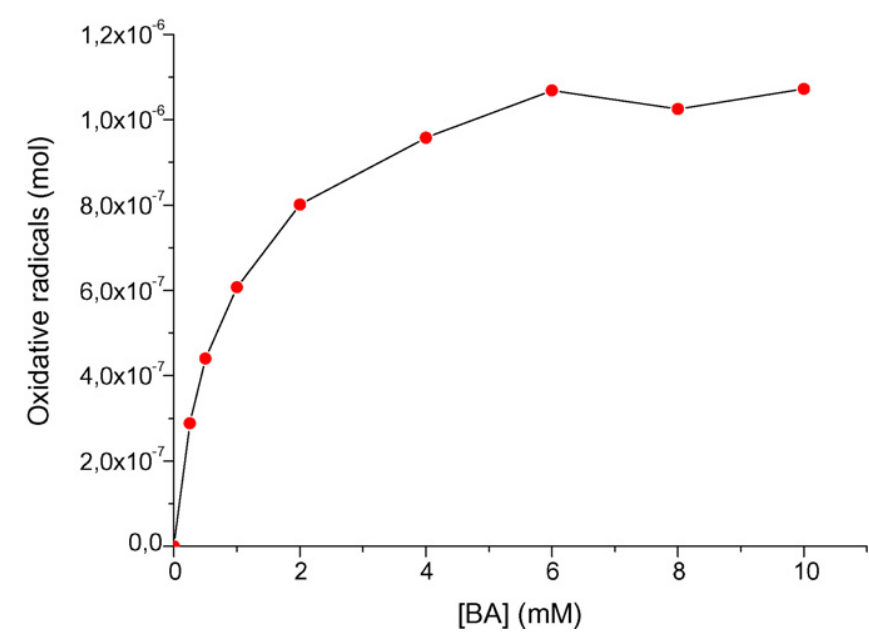

Fig. 1. Results of preliminary test: trapped oxidative radicals moles versus BA concentration.

from the linear change in the BA concentration, provided that concentrations of BA and radicals do not change significantly within the time interval (steady-state conditions). Clearly, the BA concentration should be so low that the rate of reaction of oxidative radicals with BA is negligible with respect to their rate of formation. According to Lindsey method, this condition holds true when no more than $10 \%$ of the generated radicals is trapped by benzoic acid. Looking once again at Fig. 1, this means to operate with a BA concentration equal to $0.1 \mathrm{mM}$.

\subsection{Analytical methods}

Reaction products were evaluated by high-performance liquid chromatography (HPLC) using a Hewlett-Packard 1100 liquid chromatograph equipped with a Diode Array Detector (DAD). A Nucleodur $100-5 \mathrm{C}_{18}$ EC $(5 \mu \mathrm{m}$ particle size $250 \mathrm{~mm}$ length $\times 4.6 \mathrm{~mm}$ i.d.) purchased from Macherey-Nagel, was used for all analysis. The analytes were eluted by means of a buffer solution of $50 \mathrm{mM} \mathrm{NaH} \mathrm{PO}_{4}(\mathrm{pH} \sim 7.0)$ and acetonitrile (volume ratio equal to 95:5) previously filtered with a $0.45 \mu \mathrm{m}$ membrane filter in order to eliminate all the particulates. The flowrate was set equal to $0.9 \mathrm{~mL} / \mathrm{min}$, while the oven temperature was kept to $32^{\circ} \mathrm{C}$. Then, benzoic acid and $p$-hydroxybenzoic acid were detected by absorbance at $230 \mathrm{~nm}$. The applied procedure, was taken from the Macherey-Nagel application database [21]. Two different calibration curves were built in order to correlate the [BA] or $[p-\mathrm{HBA}]$ with the peak area; the detection range was respectively $5.0 \times 10^{-6}$ to $1.0 \times 10^{-3} \mathrm{M}$ for BA and $3.4 \times 10^{-6}$ to $1.7 \times 10^{-4} \mathrm{M}$ for $p$-HBA. Further, a preliminary test was carried out to assess the possible co-elution between the BA and the $p$-HBA: this test gave a negative result since $\mathrm{BA}$ and $p$-HBA retention time were estimated equal to 2.8 and $6.8 \mathrm{~min}$ respectively.

\subsection{Design of experiments: application of Rotatable Central Composite method}

In order to minimize the number of experiments to be performed and to guarantee a certain data set statistical significance a Rotatable Central Composite method was applied. The central composite design is probably the most widely used experimental design for fitting a second-order response surface. An experimental design is rotatable if the variance of the predicted response is a function only of the distance of the experimental points from the center of the design and is not function of direction. Since the location of the
Table 1

Rotatable Central Composite operating conditions

\begin{tabular}{lllll}
\hline Test & $\mathrm{Fe}(\mathrm{II})$ coded value & $\mathrm{H}_{2} \mathrm{O}_{2}$ coded value & {$[\mathrm{Fe}(\mathrm{II})](\mathrm{mM})$} & {$\left[\mathrm{H}_{2} \mathrm{O}_{2}\right](\mathrm{mM})$} \\
\hline $\mathrm{O}$ & 0 & 0 & 0.37 & 0.60 \\
S1 & -1.41 & 0 & 0.20 & 0.60 \\
S2 & 0 & -1.41 & 0.37 & 0.20 \\
S3 & 1.41 & 0 & 0.53 & 0.60 \\
S4 & 0 & 1.41 & 0.36 & 1.00 \\
F1 & 1 & 1 & 0.48 & 0.88 \\
F2 & -1 & 1 & 0.25 & 0.88 \\
F3 & -1 & -1 & 0.25 & 0.32 \\
F4 & 1 & -1 & 0.48 & 0.32 \\
\hline
\end{tabular}

optimum is unknown prior to running the experiment, it is useful to apply a design that provides equal precision of estimation in all direction. Performing replicates of the central point allow to improve the model precision [22].

RCC method can be viewed as the combination of a 2 level factorial model $\left(2^{N}\right)$ with a Star model $(2 N+1)$ so that the minimum number of experiments to be performed $(k)$ is equal to:

$k=2^{N}+2 N+1$

where $N$ in Eq. (2) is the number of independent variables. In this case the RCC method was applied to a system of 2 independent variables (i.e. $\left[\mathrm{H}_{2} \mathrm{O}_{2}\right]$ and $\left[\mathrm{Fe}^{2+}\right]$ ) by considering two levels per variable.

The tested operating conditions, resulting from the RCC approach are reported in Table 1, and schematically shown in the RCC experimental plan shown in Fig. 2. As shown in Fig. 2, the experimental points which refer to factorial design were called F1, F2, F3, F4, whereas those of the Star design S1, S2, S3, S4. The central point was indicated as $\mathrm{O}$. In order to simplify calculations the independent variables were expressed as coded values: namely, factorial points have values of $( \pm 1, \pm 1)$, Star points were expressed as $( \pm \alpha$, $0)$ or $(0, \pm \alpha)$, while the central point had coded values of $(0,0)$. A central composite design is made Rotatable by the choice of the $\alpha$ value. Its choice depends on the number of points in the factorial part of the design. In fact, as the number of factorial points is known, as the $\alpha$ value can be estimated by applying Eq. (3):

$\alpha=n_{\mathrm{f}}^{1 / 4}$

where $n_{\mathrm{f}}$ is the number of factorial points [23]. In this study $n_{\mathrm{f}}$ was equal to four points and then Eq. (3) gave a value of 1.41 .

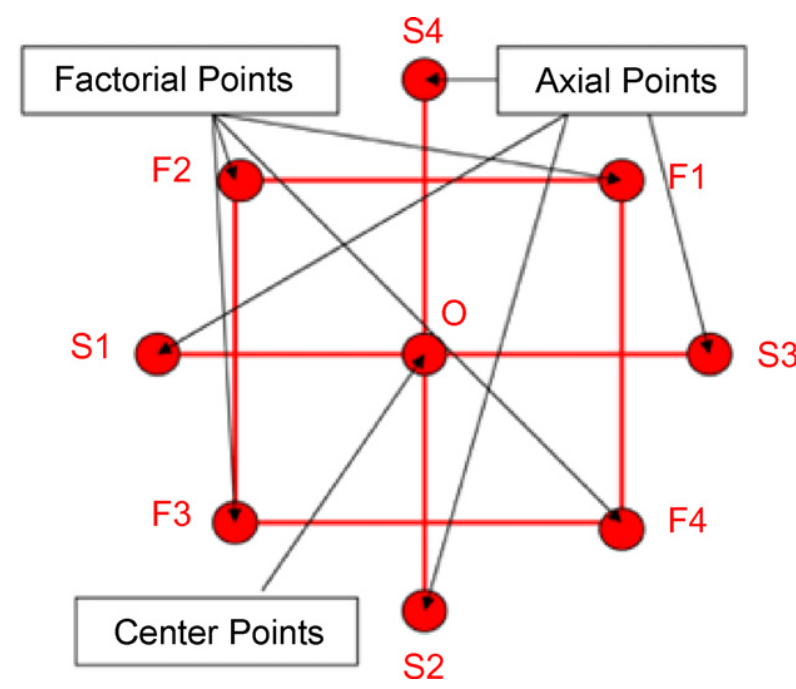

Fig. 2. Rotatable Central Composite (RCC) configuration in the experimental plan. 


\subsection{Fenton's experiments}

Fenton's reaction was carried out in both ultra-pure water and humic acid (HA) aqueous solutions with concentration set at 5, 10 and $15 \mathrm{mg} / \mathrm{L}$ in order to assess the effect of the organic matter on the oxidative radicals production. HA solutions were prepared by dissolving a known amount of humic acid sodium salt in MQ water. All the experiments were performed in $25 \mathrm{~mL}$ amber glass bottles, with a reaction volume equal to $10 \mathrm{~mL}$. The experiments for assessing the radicals total amount, were carried out in a $9 \mathrm{mM}$ BA aqueous solution at different $\mathrm{Fe}(\mathrm{II})$ and $\mathrm{H}_{2} \mathrm{O}_{2}$ concentrations (see Table 1) and $\mathrm{pH} 2.5$. The reaction time was set equal to $30 \mathrm{~min}$. The $1 \mathrm{~mL}$ samples were immediately withdrawn from the reaction environment at different reaction times between 15 and $1800 \mathrm{~s}$ and immediately quenched by adding $0.5 \mathrm{~mL}$ of 1 -propanol, whose amount was enough to compete with BA for oxidative radicals: it was assumed that this amount of added quencher had rates of reaction with oxidative radicals high enough so that, upon the addition of $\mathrm{PrOH}$, no significant reaction of BA with radicals occurred [18]. Then, the samples were filtered with $0.45 \mu \mathrm{m}$ membrane filters and analyzed by means of HPLC. All the experiments were carried out in duplicate in order to ensure data repeatability. Measurement of the oxidative radicals concentration was performed using the same procedure above described, differing only in the applied BA concentration set equal to $0.1 \mathrm{mM}$ and in the total reaction time, equal to $10 \mathrm{~min}$. The slurry-phase experiments were carried out with a quartz sand typically used as filter media in drinking water microfiltration processes. Before its use, the sand was sieved $(2 \mathrm{~mm})$ to obtain a homogeneous media, autoclaved at $120^{\circ} \mathrm{C}$ for $2 \mathrm{~min}$, allowing either sterilization and also the reduction of enzymatic activity [8]. Then, the sand was further characterized in terms of metal content, i.e. iron and manganese, according to acid digestion described in EPA method 3050B and analyzed by means of flame atomic absorption spectrometry (FLAA). The organic carbon content was determined according to Walkey-Black procedure; the sand presented $416 \mathrm{mg} / \mathrm{kg}$ of iron, $18 \mathrm{mg} / \mathrm{kg}$ of manganese, $0.34 \%$ of organic carbon and $0.59 \%$ of organic matter. The slurry-phase experiments were carried out in a range of operating conditions which are briefly summarized in Table 2; the investigated $\mathrm{H}_{2} \mathrm{O}_{2}$ and $\mathrm{Fe}(\mathrm{II})$ concentrations were the same used for the aqueous-phase experiments (see Table 1 ). In order to remove any solid residual, the samples were filtered by means of $0.45 \mu \mathrm{m}$ syringe filters before being analyzed following the procedure described before (see Section 2.3). Also in this case, the experiments were carried out in duplicate.

\section{Results and discussion}

\subsection{Aqueous-phase experiments}

\subsubsection{Measurement of the oxidative radicals total amount}

The influence of the Fenton's operating conditions, in terms of $\mathrm{Fe}(\mathrm{II})$ and $\mathrm{H}_{2} \mathrm{O}_{2}$ concentration, was assessed with respect to oxidative radicals total amount and production efficiency (i.e. molar ratio between the measured total amount of radicals and the moles of $\mathrm{H}_{2} \mathrm{O}_{2}$ initially fed to the system). Fig. 3 shows the influence of

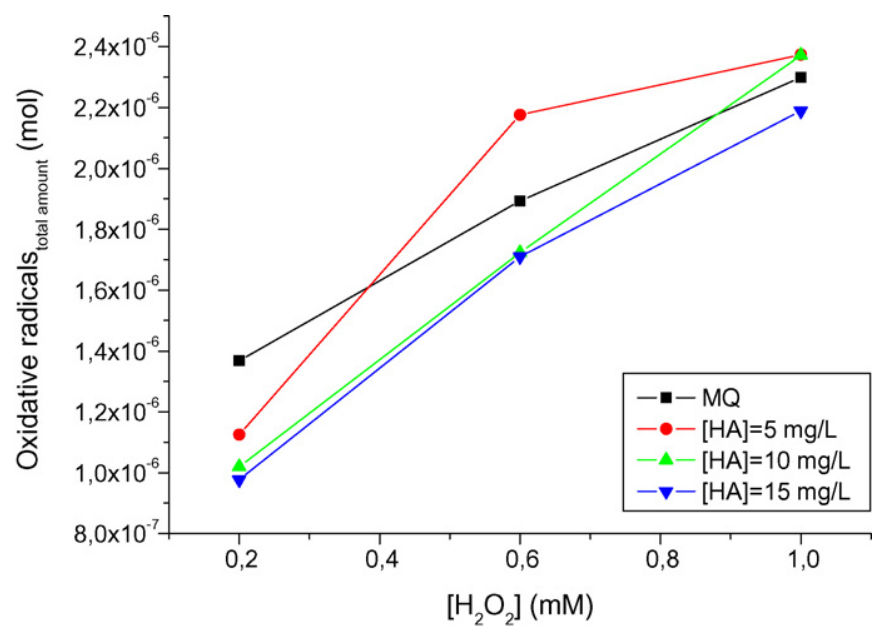

Fig. 3. Influence of the hydrogen peroxide concentration on the oxidative radicals total amount: results obtained at $[\mathrm{Fe}(\mathrm{II})]=0.365 \mathrm{mM}$.

increasing hydrogen peroxide concentration on the oxidative radicals total amount at fixed iron concentration $(0.365 \mathrm{mM})$. Within the tested concentration range, the total amount of oxidative radicals was influenced by the hydrogen peroxide concentration, with a rather linear correlation between total moles of radicals and $\mathrm{H}_{2} \mathrm{O}_{2}$ concentration. Besides, the presence of HA induced a slight decrease of the oxidative radicals total amount, even though such a decrease is also not clearly correlated to the increase of HA concentration. Anyway, the results reported in Fig. 3 confirm the expected positive influence of higher $\mathrm{H}_{2} \mathrm{O}_{2}$ concentration on the radicals production. Nevertheless, one of the main tasks in the proper design of a Fenton's process consists in maximizing the amount of oxidative radicals moles produced with respect to the amount of $\mathrm{H}_{2} \mathrm{O}_{2}$ introduced in the system, i.e. the production efficiency. As shown in Fig. 4 the production efficiency depends on the $\mathrm{Fe}(\mathrm{II}): \mathrm{H}_{2} \mathrm{O}_{2}$ molar ratio, at least up to values of this ratio around 2, where probably $\mathrm{Fe}(\mathrm{II})$ starts to act as a scavenger of hydroxyl radicals. At high $\mathrm{Fe}(\mathrm{II}): \mathrm{H}_{2} \mathrm{O}_{2}$ ratios, an important influence of the HA concentration on the oxidative radicals production efficiency was also observed. This results suggest that at these $\mathrm{Fe}(\mathrm{II}): \mathrm{H}_{2} \mathrm{O}_{2}$ ratios, competition between organic compounds and $\mathrm{Fe}(\mathrm{II})$ for hydroxyl radicals becomes important [24,25].

Looking at the results obtained for the lower $\mathrm{Fe}(\mathrm{II}): \mathrm{H}_{2} \mathrm{O}_{2}$ molar ratios, shown in Fig. 4, a decrease of the production efficiency can be noticed: in particular, for a $\mathrm{Fe}(\mathrm{II}): \mathrm{H}_{2} \mathrm{O}_{2}$ molar ratio of 0.28 the estimated production efficiency is $25 \%$, i.e. about 0.25 moles of oxidative radicals per mole of $\mathrm{H}_{2} \mathrm{O}_{2}$.

Considering the Fenton's reaction stoichiometry reported in Reactions (I) and (V)

$$
\begin{aligned}
& \mathrm{H}_{2} \mathrm{O}_{2}+\mathrm{Fe}^{2+} \rightarrow \mathrm{Fe}^{3+}+\mathrm{OH}^{-}+\mathrm{OH}^{\bullet} \\
& \mathrm{Fe}^{3+}+\mathrm{H}_{2} \mathrm{O}_{2} \rightarrow \mathrm{Fe}^{2+}+\mathrm{H}^{+}+\mathrm{HO}_{2}
\end{aligned}
$$

Table 2

\begin{tabular}{|c|c|c|}
\hline Parameter & Oxidative radicals total amount/production efficiency & Oxidative radicals concentration \\
\hline Soil:water ratio (g:g) & $1: 10,5: 10,9: 10$ & $1: 10,5: 10,9: 10$ \\
\hline $\mathrm{pH}$ & 2.5 & 2.5 \\
\hline Benzoic acid concentration (mM) & 9 & 0.1 \\
\hline Total reaction time $(\mathrm{min})$ & 30 & 10 \\
\hline Sampling time (s) & $15,30,60,120,300,600,900,1800$ & $15,30,60,120,300$ \\
\hline Quencher volume & $0.5 \mathrm{~mL} \mathrm{PrOH}$ & $0.5 \mathrm{~mL} \mathrm{PrOH}$ \\
\hline
\end{tabular}

Slurry-phase experimental setting 


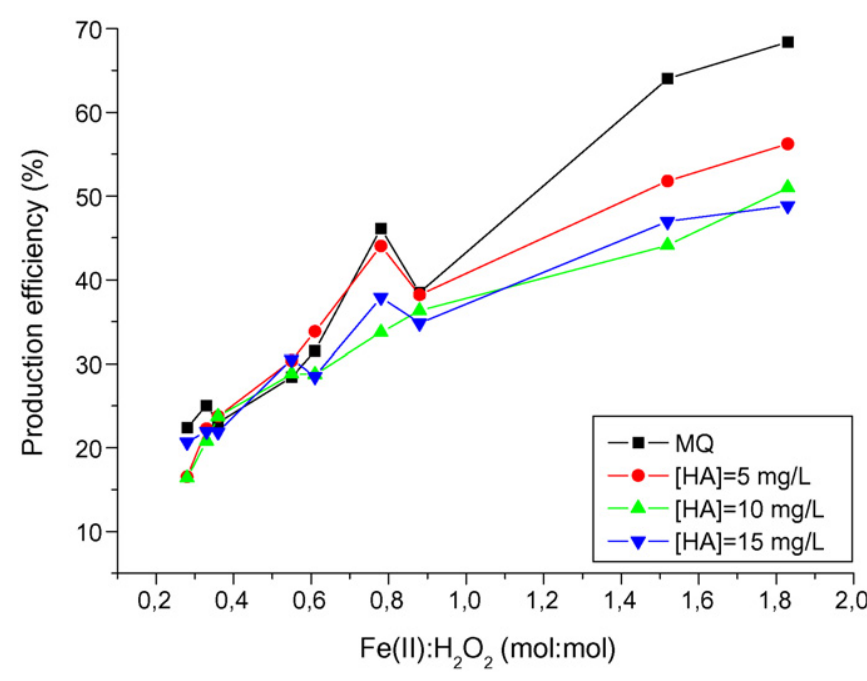

Fig. 4. Influence of the $\mathrm{Fe}(\mathrm{II}): \mathrm{H}_{2} \mathrm{O}_{2}$ molar ratio on the oxidative radical production efficiency: comparison between tests performed in ultra-pure water and with increasing amounts of humic acid (HA).

0.28 moles of $\mathrm{Fe}(\mathrm{II})$ are expected to react with 1 mole of $\mathrm{H}_{2} \mathrm{O}_{2}$ to give 0.28 moles of $\mathrm{Fe}(\mathrm{III})$ (Reaction $(\mathrm{V})$ ): the generated $\mathrm{Fe}(\mathrm{III})$ is then expected to further react with 0.28 moles $\mathrm{H}_{2} \mathrm{O}_{2}$ to produce 0.28 moles $\mathrm{Fe}(\mathrm{II})$ and other products (see Reaction (V)), as long as the $\mathrm{H}_{2} \mathrm{O}_{2}$ is completely consumed. Based on our results, it seems that the amount of oxidative radicals produced ( 0.25 moles), closely matches the number of Fe(II) moles added as reactant ( 0.28 moles). This suggests that the expected recycle of $\mathrm{Fe}(\mathrm{III})$ to $\mathrm{Fe}(\mathrm{II})$ through Reaction (V) may not take place under the tested operating condition, thus stopping the radicals propagation cycle. Such conversion may be hindered for different reasons. It has been reported that the generation rate of $\mathrm{Fe}(\mathrm{II})$ is much lower than its consumption rate; thus, the amount of re-converted $\mathrm{Fe}(\mathrm{II})$ could be very low unless other compounds, such as hydroquinone-like intermediates or cathecols, which are capable of quickly reducing $\mathrm{Fe}(\mathrm{III})$ to $\mathrm{Fe}(\mathrm{II})$, are present [26]. Besides, organic acids such as maleic and oxalic acid, might scavenge $\mathrm{Fe}(\mathrm{III})$ during the process by forming stable complexes (e.g. ferrioxalate) which are not readily available to react with $\mathrm{H}_{2} \mathrm{O}_{2}$ through Reaction (V) $[19,25]$.

The above explanation would be true if only hydroxyl radicals were present in the Fenton's system. Since also other oxidative radicals are generally present in Fenton's system, it can be also argued that the fraction of oxidative radicals represented by hydroxyl radicals reacts through Reaction (II), thus producing less reactive perhydroxyl radicals, which have a less effective reaction with the probe compound.

\subsubsection{Measurement of the oxidative radicals concentration versus time}

As general result during this set of experiments, it was noticed that whichever concentration of $\mathrm{H}_{2} \mathrm{O}_{2}$ and $\mathrm{Fe}(\mathrm{II})$ was tested within the experimental plan, oxidative radicals concentration decreased of approximately $80 \%$ within 5 min from the addition of $\mathrm{H}_{2} \mathrm{O}_{2}$ (data not shown). This suggests that oxidative radicals production, due to hydrogen peroxide reaction, takes place in the very first seconds of the process, followed by a quite rapid radical consumption. The influence of the Fenton's operating conditions on oxidative radicals concentration was evaluated using, as performance indicator, the average radicals concentration: its value was derived from the data measured during the experiments at fixed times. The effect of increasing organic matter content was further investigated by plotting the oxidative radical concentration versus the HA:Fe(II) weight

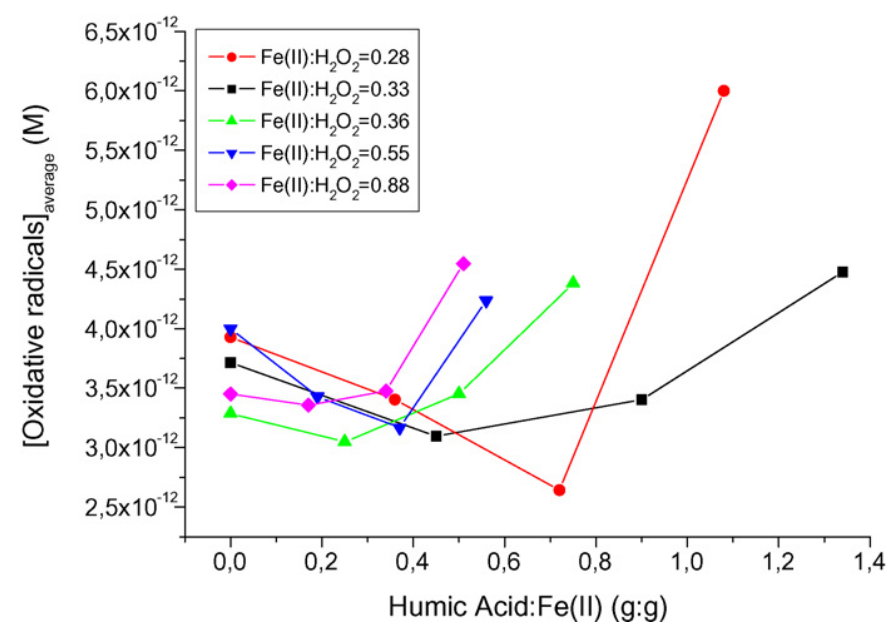

Fig. 5. Effect of the HA:Fe(II) (humic acid to ferrous ions weight ratio) on the oxidative radicals concentration: comparison between different $\mathrm{Fe}(\mathrm{II}): \mathrm{H}_{2} \mathrm{O}_{2}$ molar ratios

ratio as shown in Fig. 5. Although the effect of this ratio on the radicals concentration is not so important, the obtained results suggest that an increase of HA:Fe(II) ratio has a detrimental effect on oxidative radicals concentration for low $\mathrm{HA}: \mathrm{Fe}(\mathrm{II})$ ratios, whereas when such a ratio increases to values around 1 or higher, the influence becomes positive. This may suggest that under given operating conditions, the organic matter may play a role in the decomposition of $\mathrm{H}_{2} \mathrm{O}_{2}$, thus increasing the radical concentration [27].

\subsection{Slurry-phase experiments}

The results reported in Fig. 6 clearly indicate that whichever the soil:water ratio, the radicals generated in a soil slurry system are up to one order of magnitude lower than those measured in ultra-pure water (MQ). Besides, it is worth pointing out that the soil:water ratio has a weak influence on the radicals production efficiency. A positive correlation between the production efficiency of oxidative radicals and $\mathrm{Fe}(\mathrm{II}): \mathrm{H}_{2} \mathrm{O}_{2}$ molar ratio was also noted in slurry-phase experiments. As far as the effect of the $\mathrm{Fe}(\mathrm{II}): \mathrm{H}_{2} \mathrm{O}_{2}$ ratio on the oxidative radicals concentration is concerned, Fig. 7 clearly shows that in slurry-phase experiments the radicals concentration was always almost one order of magnitude lower than in aqueous phase

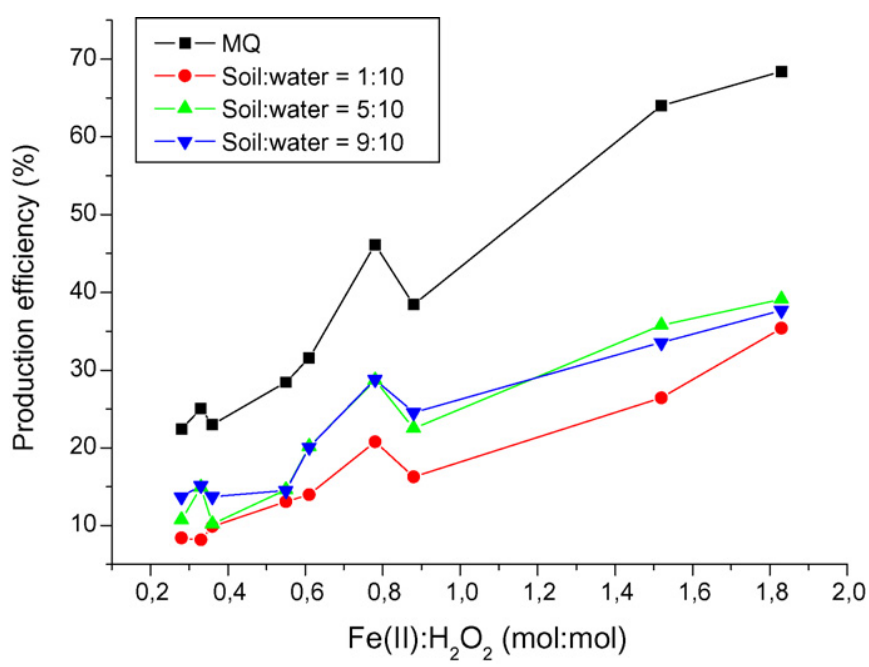

Fig. 6. Influence of the $\mathrm{Fe}(\mathrm{II}): \mathrm{H}_{2} \mathrm{O}_{2}$ molar ratio on the production efficiency of oxidative radicals: comparison between $\mathrm{MQ}$ data and slurry tests at different soil:water ratios. 


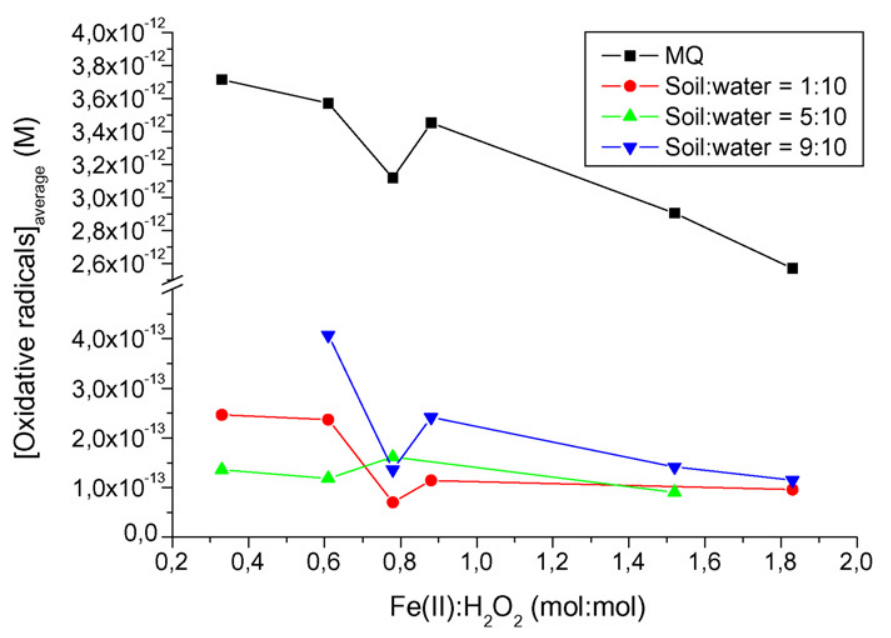

Fig. 7. Influence of the $\mathrm{Fe}(\mathrm{II}): \mathrm{H}_{2} \mathrm{O}_{2}$ molar ratio on oxidative radicals concentration: comparison between MQ data and slurry tests at different soil:water ratios.

experiments. This means that the presence of the soil matrix has a detrimental effect on both amount and concentration of radicals available for oxidizing organic compounds, whereas it probably drives hydrogen peroxide decomposition through other pathways, such as disproportion reactions. This effect, usually accounted for in in situ Fenton' s application by increasing the hydrogen peroxide concentration with respect to the one used in water treatment, clearly depends on the properties of the soil matrix. The coarse sand used in this paper probably did not greatly affect the radical production, due to its low specific surface area. A different behaviour would probably be observed in real soils, whose texture is characterized by a rather high fraction of fine particles that may have a more effective role in decomposing hydrogen peroxide to other products than oxidative radicals.

\section{Conclusions}

The application of an indirect method to quantify oxidative radicals allowed to evaluate the effect of different operating conditions on the total amount of radicals generated, their production efficiency (i.e. $\mathrm{H}_{2} \mathrm{O}_{2}$ to radicals conversion with respect to the stoichiometric one) and finally on the fate of oxidative radicals (i.e. concentration and lifetime in the reaction environment) in aqueous and soil slurry Fenton's systems.

Within the tested operating conditions, the $\mathrm{Fe}(\mathrm{II}): \mathrm{H}_{2} \mathrm{O}_{2}$ ratio was observed to affect the oxidative radicals production efficiency, which achieved a maximum $70 \%$ value for the highest tested $\mathrm{Fe}(\mathrm{II}): \mathrm{H}_{2} \mathrm{O}_{2}$ ratio. At low $\mathrm{Fe}(\mathrm{II}): \mathrm{H}_{2} \mathrm{O}_{2}$ molar ratios, the production efficiency was particularly low, suggesting that either $\mathrm{Fe}(\mathrm{II})$ was unable to complete the redox cycle generally supposed to occur during a Fenton's reaction or the hydroxyl radicals mostly reacted with hydrogen peroxide forming less reactive perhydroxyl radicals. Moreover, the organic matter content was observed to negatively affect the oxidative radicals production efficiency, especially for the higher $\mathrm{Fe}(\mathrm{II}): \mathrm{H}_{2} \mathrm{O}_{2}$ molar ratios.

Despite some weak effects were noticed and discussed, the influence of dissolved organic matter on the radicals production was of minor importance. On the contrary, a one order of magnitude reduction in both radicals amount generated and concentration was observed when soil was added to the reaction environment.

It is worth pointing out that the results discussed in this paper were obtained on a model system, where a simple silica sand was used as soil matrix. Besides, also the operating conditions selected for this study were those typically used in aqueous Fenton's sys- tems. These choices were made in order to obtain a simplified experimental system, since no literature data on the quantification of oxidative radicals in soil slurry systems were available. Nevertheless, most of the effects that can play an important role in real world Fenton's applications were obviously not accounted for. Therefore, further research is needed to assess the feasibility of the oxidative radicals quantification method also for this kind of systems.

\section{Acknowledgements}

The authors wish to acknowledge Dr. Luigi Galeotti and Dr. Gloria Carucci of the Laboratory of Sanitary-Environmental Engineering of the Department of Hydraulics, Transportations and Roads of "Sapienza" University of Rome for their effective collaboration during the soil characterization.

\section{References}

[1] J. Kiwi, A. Lopez, V. Nadtochenko, Mechanism and kinetics of the OH-radical intervention during Fenton oxidation in the presence of a significant amount of radical scavenger $\left(\mathrm{Cl}^{-}\right)$, Environ. Sci. Technol. 34 (2000) 2162-2168.

[2] R.J. Watts, A.M. Teel, Chemistry of modified Fenton's reagent (catalyzed $\mathrm{H}_{2} \mathrm{O}_{2}$ propagations-CHP) for in situ soil and groundwater remediation, J. Environ. Eng. ASCE 131 (2005) 612-622.

[3] A.L. Teel, R.J. Watts, Degradation of carbon tetrachloride by modified Fenton's reagent, J. Hazard. Mater. B94 (2002) 179-189.

[4] B.A. Smith, A.L. Teel, R.J. Watts, Mechanism for the destruction of carbon tetrachloride and chloroform DNAPLs by modified Fenton's reagent, J. Contam. Hydrol. 85 (2006) 229-246.

[5] R.J. Watts, M.K. Foget, S.-H. Kong, A.L. Teel, Hydrogen peroxide decomposition in model subsurface systems, J. Hazard. Mater. B69 (1999) 229-243.

[6] A.L. Teel, C.R. Warberg, D.A. Atkinson, R.J. Watts, Comparison of mineral and soluble iron Fenton's catalysts for the treatment of trichloroethylene, Water Res. 35 (4) (2001) 977-984.

[7] W.P. Kwan, B.M. Voelker, Decomposition of hydrogen peroxide and organic compounds in the presence of dissolved iron and ferrihydrite, Environ. Sci. Technol. 36 (2002) 1467-1476.

[8] W.P. Kwan, B.M. Voelker, Rates of hydroxyl radical generation and organic compound oxidation in mineral-catalyzed Fenton-like systems, Environ. Sci. Technol. 37 (2003) 1150-1158.

[9] R.L. Valentine, H.C.A. Wang, Iron oxide surface catalyzed oxidation of quinoline by Hydrogen peroxide, J. Environ. Eng. 124 (1998) 31-38.

[10] S.-S. Lin, M.D. Gurol, Catalytic decomposition of hydrogen peroxide on iron oxide: kinetics, mechanism and implications, Environ. Sci. Technol. 32 (10) (1998) 1417-1423.

[11] M. Arienzo, J. Chiarenzelli, R. Scrudato, J. Pagano, L. Falanga, B. Connor Iron-mediated reactions of polychlorinated biphenyls in electrochemical peroxidation process (ECP), Chemosphere 44 (2001) 1339-1346.

[12] J.R. Chiarenzelli, R.J. Scrudato, M.L. Wunderlich, J. Pagano, Combined steam distillation and electrochemical peroxidation (ECP) treatment of river sediment contaminated by PCBs, Chemosphere 45 (2001) 1159-1165.

[13] R. Baciocchi, M.R. Boni, L. D’Aprile, Hydrogen peroxide lifetime as an indicator of the efficiency of 3-chlorophenol Fenton's and Fenton-like oxidation in soils, J. Hazard. Mater. B96 (2003) 305-329.

[14] R. Baciocchi, M.R. Boni, L. D'Aprile, Application of $\mathrm{H}_{2} \mathrm{O}_{2}$ lifetime as an indicator of TCE Fenton-like oxidation in soils, J. Hazard. Mater. B107 (2004) 97102.

[15] C. Tai, J.-F. Peng, J.-F. Liu, G.-B. Jiang, H. Zou, Determination of hydroxyl radicals in advanced oxidation processes with dimethyl sulfoxide trapping and liquid chromatography, Anal. Chim. Acta 527 (2004) 73-80.

[16] J.-F. Jen, M.-F. Leu, T.C. Yang, Determination of hydroxyl radicals in an advanced oxidation process with salicylic acid trapping and liquid chromatography, J. Chromatogr. A 796 (1998) 283-288.

[17] E. Kilinc, Determination of the hydroxyl radical by its adduct formation with phenol and liquid chromatography/electrochemical detection, Talanta 65 (2005) 876-881.

[18] M.E. Lindsey, M.A. Tarr, Quantitation of hydroxyl radical during Fenton oxidation following a single addition of iron and peroxide, Chemosphere 41 (2000) 409-417.

[19] J.F. Rivas, F.J. Beltran, J. Frades, P. Buxeda, Oxidation of $p$-hydroxybenzoic acid by Fenton's reagent, Water Res. 35 (2) (2001) 387-396.

[20] X.Zhou, K. Mopper, Determination of photochemically produced hydroxyl radicals in seawater and freshwater, Mar. Chem. 30 (1990) 71-78.

[21] Separation of unsaturated carboxylic acids, Macherey-Nagel application no. 110670. Available on www.mn-net.com.

[22] P.W. Araujo, R.G. Brereton, Experimental design II. Optimization, Trends Anal. Chem. 15 (2) (1996) 63-70. 
[23] D.C. Montgomery, Design and Analysis of Experiments, third ed., John Wiley and Sons, New York, 1980, ISBN 0-471-52000-4

[24] E. Neyens, J. Baeyens, A review of classic Fenton's peroxidation as an advanced oxidation technique, J. Hazard. Mater. B98 (2003) 33-50.

[25] Y. Du, M. Zhou, L. Lei, Kinetic model of 4-CP degradation by Fenton $/ \mathrm{O}_{2}$ system, Water Res. 41 (5) (2007) 1121-1133.
[26] Y. Du, M. Zhou, L. Lei, The role of oxygen in the degradation of $p$-chlorophenol by Fenton system, J. Hazard. Mater. B139 (2007) 108-115.

[27] L.L. Bissey, J.L. Smith, R.J. Watts, Soil organic matter-hydrogen peroxide dynamics in the treatment of contaminated soils and groundwater using catalyzed $\mathrm{H}_{2} \mathrm{O}_{2}$ propagations (modified Fenton's reagent), Water Res. 40 (2006) 2477-2484. 